\title{
Expression of miRNAs involved in phosphate homeostasis and senescence is altered in glyphosate-treated maize
}

\author{
Marek Żywicki ${ }^{1}$ Joanna Gracz ${ }^{2} \cdot$ Wojciech Karłowski $^{1} \cdot$ Tomasz Twardowski $^{2}$ • \\ Agata Tyczewska ${ }^{2}$
}

Received: 22 July 2015/Revised: 12 November 2015/Accepted: 13 November 2015/Published online: 25 November 2015

(C) The Author(s) 2015. This article is published with open access at Springerlink.com

\begin{abstract}
Glyphosate is a systemic, nonselective and most widely used herbicide in the world. The introduction of glyphosate-resistant crops in the mid-1990s resulted in a dramatic increase in the use of glyphosate herbicide making it most widely used herbicide in the world. The average maize yield loss in the field caused by pests is around $20 \%$ but in many regions it is much higher. It is now clear that glyphosate causes broader range of physiological alterations than previously assumed and some plants gain higher level of resistance to glyphosate without the need to use genetic engineering methods. To understand the mechanisms of such heightened resistance we must first
\end{abstract}

Communicated by P. Wojtaszek.

Electronic supplementary material The online version of this article (doi:10.1007/s11738-015-2022-5) contains supplementary material, which is available to authorized users.

Agata Tyczewska

agatat@ibch.poznan.pl

Marek Żywicki

marek.zywicki@amu.edu.pl

Joanna Gracz

j.gracz@gmail.com

Wojciech Karłowski

wmk@amu.edu.pl

Tomasz Twardowski

twardows@ibch.poznan.pl

1 Department of Computational Biology, Faculty of Biology, Institute of Molecular Biology and Biotechnology, Adam Mickiewicz University in Poznań, Umultowska 89, 61-614 Poznań, Poland

2 Department of RNA Biology, Institute of Bioorganic Chemistry, Polish Academy of Sciences, Noskowskiego 12/14, 61-704 Poznań, Poland know the processes mediating the plants' death in response to glyphosate treatment. Here, we show that 12 miRNAs, belonging to miR167, miR396, miR159, miR156, miR169, miR444 and miR827 families, are significantly upregulated, and one, miR166, downregulated following glyphosate treatment. These miRNAs have been previously shown to be involved in abiotic stress responses and implicated in senescence. Strikingly, two of the induced miRNAs, miR444 and miR827, have been shown to regulate phosphate transport pathways, which seem to be common for Pi and glyphosate uptake.

Keywords Maize - Herbicidal stress - Glyphosate miRNAs · Pi homeostasis - Senescence

\section{Introduction}

Glyphosate, a glycine derivative ( $N$-(phosphonomethyl)glycine), is a broad-spectrum, post-emergence herbicide. Concurrently with the release of genetically engineered glyphosate-resistant soybean in 1996, there was a dramatic increase in the use of glyphosate; it has now become the most widely used herbicide in the world. Glyphosate is active as a salt and effective only when it comes into contact with green, growing parts of plants (Duke and Powles 2008) after which it becomes distributed throughout the organism. There appear to be at least two mechanisms of glyphosate uptake (active and passive) in plants; however, they are not well understood to date. An active system operates at low concentrations of herbicide and may presumably involve a phosphate transporter since it has been shown that the glyphosate uptake is inhibited by sodium phosphate and phosphonoformic acid, competitive inhibitors of phosphate assimilation in plant cells (Denis 
and Delrot 1993; Hetherington et al. 1998; Morin et al. 1997; Shaner 2009). The interruption of essential metabolic pathways manifests as yellowing and wilting of the plant leaves followed by browning. Concurrently glyphosate induces decomposition of the plant's roots and rhizomes. Plants sprayed with glyphosate die within 10-14 days. The symptoms resulting from glyphosate administration resemble those occurring during senescence. Leaf senescence is manifested by a tissue-specific changes like chloroplasts degeneration and disintegration of photosynthetic apparatus. The senescence hypothesis, known as the nutrient relocalization hypothesis, states that annual plants at the end of growing season face limited supplies of micro- and macronutrients and therefore relocate resources such as copper, phosphate and nitrate into vegetative tissues to enhance seed yields (Gregersen et al. 2008; Himelblau and Amasino 2001). Interestingly, large-scale expression profiling experiments indicate the existence of cross-talk between developmental senescence and responses to abiotic stresses (Allu et al. 2014; Buchanan-Wollaston et al. 2005).

Administration of herbicide Roundup ${ }^{\circledR}$ to plant causes glyphosate-mediated inhibition of the 5-enolpyruvylshikimate-3-phosphate synthase (EPSPS) resulting in the disruption of synthesis of aromatic amino acids and their derivatives (Duke and Powles 2008). However, some studies dispute that glyphosate toxic effect is strictly linked to EPSPS inhibition; it has not been verified that a depletion in concentration of aromatic amino acids occurs in glyphosate-treated plants (Serra et al. 2013). Instead, acting as a metal chelator, glyphosate could negatively contribute to the activity of many enzymes, altering plant physiological processes such as photosynthesis, carbon metabolism, mineral nutrition and oxidative events (Geiger et al. 1986; Orcaray et al. 2012; Romero et al. 2011).

The major drawback of wide use of herbicides is a continuous selection of plants able to both survive and reproduce under herbicide treatment. As a consequence, plants with improved survival properties are able to become dominant and distributed over large areas (Menne and Köcher 2008). There are currently 447 unique cases among 244 species (142 dicots and 102 monocots) of herbicide resistant weeds. Strikingly, they cover 22 of the 25 known mechanisms of herbicide action (156 different herbicides) (Heap 2015). The development of resistance to herbicides, however, is not restricted to weeds. Maize, Zea mays, is one of the world's most cultivated crop plants. Its great economic potential is shown in its broad base use in food, fodder and textile industry as well as in bioethanol production. Unexpectedly the value of maize crop is nowadays threatened by its susceptibility to chemicals used for weeds and pests control. The average pest-induced yield loss of maize in the field is around $20 \%$, and often much higher. Field observation has shown that some of maize lines are more sensitive than others to herbicide spraying (K. Adamczewski, J. Adamczyk, personal communication).

Plants, as well as other organisms, have employed gene expression regulation by small RNA molecules in addition to regulatory DNA sequences and different types of regulatory proteins to achieve precise and timely regulation of stress-responsive gene networks. Small non-coding RNAs, discovered two decades ago, not only regulate gene expression on post-transcriptional and transcriptional levels, but also affect the organization and modification of chromatin. Moreover, they regulate growth and development of organisms, organ development, hormone signaling, and defense against pathogens among other physiological functions (Moazed 2009). They also function during environmental stress adaptation, providing precise regulation of gene expression. In plants small RNAs exhibit unexpected complexity, however, two main categories are distinguished based on their biogenesis and function. These are miRNAs (microRNAs) and siRNAs (short interfering RNAs).

To date little is known about the involvement of miRNAs in response to herbicide treatment in plants. Here, we describe the roles of miRNA in late response to one of the most commonly used herbicide worldwide-glyphosate. We performed the miRNA expression profiling in maize leaves 7 days after glyphosate administration, which is a crucial time point for selection of the death or survival pathway.

\section{Materials and methods}

\section{Plant material}

For analyses we used maize inbred line S79757, seeds were obtained from a local breeder HR Smolice. We chose maize lines based on field tests conducted to verify the response of 25 maize inbred lines to Roundup ${ }^{\circledR}$ stress (K. Adamczewski, data not published). Based on these tests we chose the line S79757 as it showed the most prominent response to herbicide stress. Maize seedlings were grown in a greenhouse in controlled temperature $\left(22{ }^{\circ} \mathrm{C}\right)$, humidity and light conditions (16/8 h, light/dark). Uniform-sized seedlings were selected and divided into two groups. The experimental group was sprayed with a sublethal dose of herbicide Roundup ${ }^{\circledR}$ (1.0 1/ha, $300 \mathrm{~g}$ glyphosate) with adjuvant AS $500 \mathrm{SL}$ (4.0 1/ha) 2 weeks after plants' emergence (at the 4-5 leaf), the control group was cultured without Roundup ${ }^{\circledR}$ treatment. Leaves were harvested 7 days after Roundup ${ }^{\circledR}$ application, to evaluate the late response to stress conditions. Leaves were harvested at 
the same times for both experimental and control plants. Plant material samples were immediately frozen in liquid nitrogen and stored at $-80{ }^{\circ} \mathrm{C}$.

\section{RNA isolation, cDNA library preparation and sequencing}

The small RNA fractions $<200 \mathrm{nt}$ were isolated from $250 \mathrm{mg}$ of leaves collected and pooled from six plants. Isolation was performed using mirVana miRNA Isolation Kit (Ambion) following the manufacturer's instructions. RNA concentration was measured with Nanodrop 2000 and the quality was determined with Bioanalizator 2100 (Agilent; Small RNA Kit). cDNA libraries were prepared for sequencing with TruSeq SmallRNA Preparation Kit (Illumina), this included the following steps: ligation of adapters, RT-PCR amplification, gel purification of small RNA libraries and validation. Genome Analyzer IIx (Illumina) was used for $72 \mathrm{nt}$ single-read sequencing.

\section{Analysis of sequencing data}

Raw sequencing reads were examined for quality using FastQC software. Next, $3^{\prime}$ adaptor sequences were trimmed using cutadapt tool (Martin 2011). Only sequences with removed adaptors and resulting length of 18-24 nt were selected for downstream analysis. After adaptor removal, library quality was examined with FastQC software to verify that no further quality filtering or trimming was necessary. In order to enable identification of conserved miRNAs not observed previously in maize, the non-redundant subset of Magnoliophyta miRNAs obtained from miRBase (release 21) (Kozomara and Griffiths-Jones 2014) was used as reference miRNA sequences. This was prepared by clustering miRBase sequences with cdhit-est program (sequence identity $100 \%$, minimum overlap $80 \%$ ) (Fu et al. 2012). Maize miRNA was selected as the cluster representative, if present within a cluster. Expression profiling was performed with quantifier.pl script from the mirdeep2 package (Friedländer et al. 2012) with one mismatch between read and reference allowed. The high confidence set of expressed miRNAs was constructed by selection of miRNAs present in minimum of two samples with at least 10 reads. The resulting table of raw miRNA read counts is available in the Supporting Information section (Table S1).

Differential gene expression analysis was performed using $\mathrm{R}$ statistical environment and edgeR package (Robinson et al. 2010). For miRNA target prediction, the psRNA Target web server (Dai and Zhao 2011) was used. Functional annotation of the target mRNAs was done using the in-house script based on information downloaded from Plant Ensembl portal. For the Gene Ontology enrichment analysis the g:Profiler web server (Reimand et al. 2011) was used.

\section{Results}

\section{cDNA libraries}

The estimation of miRNA expression levels by high throughput sequencing has been shown to be one of the most reliable methods available. To ensure a proper estimation of miRNA expression levels we used two replicates for both, control and glyphosate treated samples (Fig S1). After pre-processing the sequence reads, we found that the most prominent length of the reads was 24 nt (Fig. 1a) in all four libraries. In plants, 24 nt long RNAs are usually derived from transposable elements and repetitive sequences and function as guide RNAs in transcriptional silencing (TGS) (Baev et al. 2010). However, only a small number of reads from our cDNA libraries (1-3\%) could be mapped to the collection of maize repetitive elements obtained from RepBase (Jurka 2000). Instead, the vast majority of $24 \mathrm{nt}$ long RNAs were found to be derived from ribosomal RNAs. They play a critical role in control of rRNA dosage in hybrid and polyploid plants by driving methylation of rRNA loci [for review see Preuss and Pikaard (2007)]. We did not, however, observe any significant glyphosate treatment-dependent changes in the amounts of rRNA-derived 24 nt long RNA class.

We found that $21 \mathrm{nt}$ was the most prominent length of sequence reads from our experiments that mapped to miRNAs (Fig. 1b); this size is canonical for plant miRNAs. With few exceptions, this general trend was also observed for most of the analyzed individual miRNA length distributions.

\section{Novel conserved miRNAs in maize}

According to miRBase (release 21), there are 321 known miRNAs in maize. This number is relatively low compared to that found in other closely related plant species, e.g., Oryza sativa with 713 known miRNAs. Our objective was to identify known as well as novel miRNA species expressed in S79757 maize line in either control conditions or after glyphosate treatment. We did this by aligning short sequencing reads to the complete set of known miRNAs from Magnoliophyta (6547 precursor and 7956 mature miRNA sequences). We considered the sequence reads which did not reveal similarity to known maize miRNAs, but instead were mapped to precursor regions overlapping with positions of known miRNAs from other organisms as novel conserved maize miRNAs. In this way we identified 13 miRNAs that have been 
A

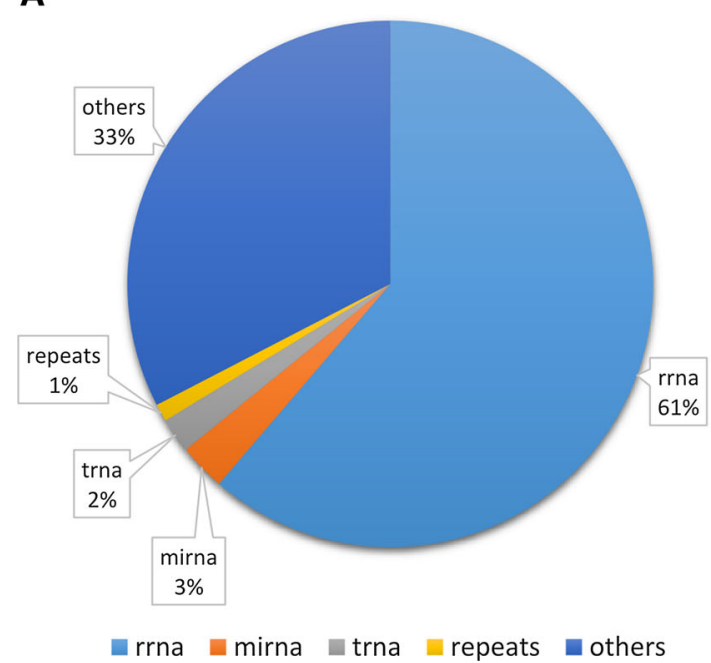

Fig. 1 Composition of cDNA libraries. a The distribution of 20-25 nt long fragments within the sequenced cDNA libraries. The largest uniform group represents rRNAs (61\%), miRNAs constitute the next biggest group (3\%), then tRNAs $(2 \%)$ and repeats $(1 \%)$. Unclassified small RNAs (others) constitute $33 \%$ of total reads. b The

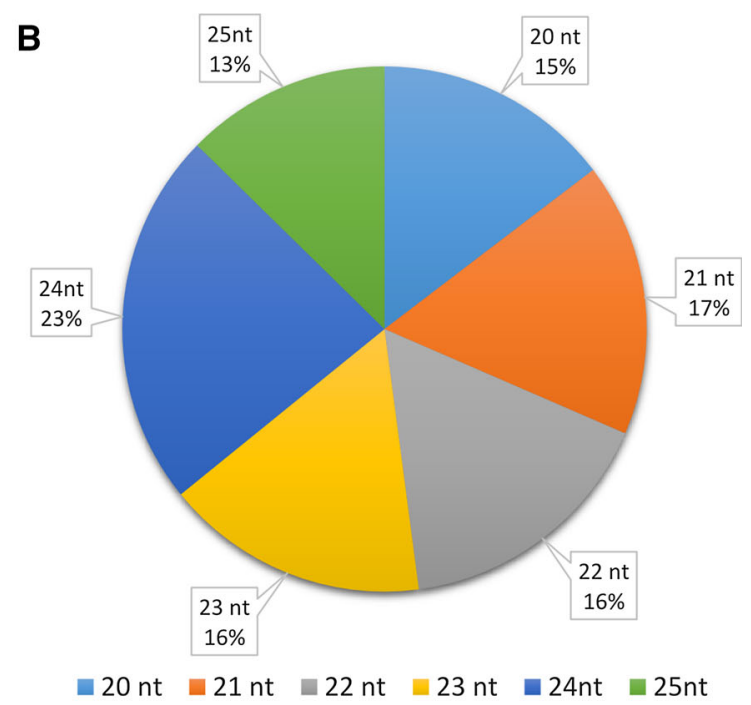

distribution of fragments in cDNA libraries based on their length. The most abundant group is the $24 \mathrm{nt}$ (siRNAs) making up $23 \%$ of total, followed by $21 \mathrm{nt}$ (miRNAs) making up $17 \%$ of total. Small RNAs of other sizes: 23, 22, 20 and 25 nt make up 16, 16, 15 and $13 \%$ of total reads, respectively

Table 1 Novel maize miRNAs identified in this study

\begin{tabular}{lllll}
\hline miRNA ID & $\begin{array}{l}\text { Number of } \\
\text { supporting reads }\end{array}$ & $\begin{array}{l}\text { Number of } \\
\text { supporting libraries }\end{array}$ & $\begin{array}{l}\text { Novel/known maize } \\
\text { miRNA family }\end{array}$ & Sequence $\left(5^{\prime}-3^{\prime}\right)$ \\
\hline gma-miR4995 & 45 & 4 & Novel & AGGCAGTGGCTTGGTTAAGGG \\
stu-miR479 & 12 & 4 & Novel & TGAGCCGAACCAATATCACTC \\
gma-miR6300 & 17 & 3 & Novel & GTCGTTGTAGTATAGTGG \\
ptc-miR6478 & 129 & 4 & Novel & CCGACCTTAGCTCAGTTGGTG \\
ath-miR8175 & 51 & 4 & Novel & GATCCCCGGCAACGGCGCCA \\
ppe-miR167c & 258 & 4 & Known & TGAAGCTGCCAGCATGATCTGA \\
tae-miR167c-5p & 131 & 4 & Known & TGAAGCTGCCAGCATGATCTGC \\
vvi-miR159c & 226 & 4 & Known & TTTGGATTGAAGGGAGCTCTA \\
osa-miR444b.1 & 11 & 4 & Known & TGTTGTCTCAAGCTTGCTGCC \\
tcc-miR169i & 11 & 4 & Known & TAGCCAAGGATGAGTTGCCTG \\
stu-miR408b-3p & 189 & 4 & Known & TGCACTGCCTCTTCCCTGGCT \\
ata-miR398f-5p & 11 & 3 & Known & GGGTCGAACTGAGAACACATG \\
vvi-miR398b & 11 & 3 & Known & TGTGTTCTCAGGTCGCCCCTG \\
\hline
\end{tabular}

Maize miRNAs "novel" stand for not previously described in maize miRNAs, "known" represent previously not described miRNAs that can be assigned to already known maize miRNA family. Number of supporting reads describes the total number of reads for a particular sequence in all sequenced cDNA libraries. Number of supporting libraries describes the number of libraries in which a particular miRNA has been identified gma Glycine max, stu Solanum tuberosum, ptc Populus trichocarpa, ath Arabidopsis thaliana, ppe Prunus persica, tae Triticum aestivum, vvi Vitis vinifera, osa Oryza sativa, tcc Theobroma cacao, ata Aegilops tauschii

shown to be present in other plants, but were not reported previously in maize (Table 1). Interestingly, eight of those are novel members of previously identified maize miRNA families that show minor sequence heterogeneity mostly outside of the seed region (Fig. 2). The remaining five novel miRNAs identified represent families not observed previously in maize. Although members of those miRNA families are usually found widespread in plants, the occurrence of molecules with identical sequence seen in maize was limited to one to three other species. The number of sequence reads that identify novel miRNAs differ from 12 for stu-miR479 up to 129 for ptc-miR6478; however, in all cases the mature miRNAs were observed in at least three out of four sequenced cDNA libraries (two control and two treated replicates) supporting reliability of these observations. 
Fig. 2 Sequence alignments of maize miRNA families with novel identified members. Figure shows sequence alignments of homologous (novel in maize) small RNAs (bolded) already described in other species with known members of maize miRNA families. zma Zea mays, ppe Prunus persica, tae Triticum aestivum, tcc Theobroma cacao, ata Aegilops tauschii, vvi Vitis vinifera, osa Oryza sativa, stu Solanum tuberosum

$\begin{aligned} \text { ppe-miR167c } & \text { TGAAGCTGCCAGCATGATCTGA } \\ \text { tae-miR167c-5p } & \text { TGAAGCTGCCAGCATGATCTGC } \\ \text { zma-miR167a-5p } & \text { TGAAGCTGCCAGCATGATCTA } \\ \text { zma-miR167b-5p } & \text { TGAAGCTGCCAGCATGATCTA } \\ \text { zma-miR167d-5p } & \text { TGAAGCTGCCAGCATGATCTA } \\ \text { zma-miR167c-5p } & \text { TGAAGCTGCCAGCATGATCTA } \\ \text { zma-miR167e-5p } & \text { TGAAGCTGCCAGCATGATCTG } \\ \text { zma-miR167f-5p } & \text { TGAAGCTGCCAGCATGATCTG } \\ \text { zma-miR167g-5p } & \text { TGAAGCTGCCAGCATGATCTG } \\ \text { zma-miR167h-5p } & \text { TGAAGCTGCCAGCATGATCTG } \\ \text { zma-miR167i-5p } & \text { TGAAGCTGCCAGCATGATCTG } \\ \text { zma-miR167j-5p } & \text { TGAAGCTGCCAGCATGATCTG } \\ & \\ \text { tcc-miR169i } & \text { TAGCCAAGGATGAGTTGCCTG-- } \\ \text { zma-miR169a-5p } & \text { CAGCCAAGGATGACTTGCCGA-- } \\ \text { zma-miR169b-5p } & \text { CAGCCAAGGATGACTTGCCGA-- } \\ \text { zma-miR169c-5p } & \text { CAGCCAAGGATGACTTGCCGG-- } \\ \text { zma-miR169f-5p } & \text { TAGCCAAGGATGACTTGCCTA-- } \\ \text { zma-miR169g } & \text { TAGCCAAGGATGACTTGCCTA-- } \\ \text { zma-miR169h } & \text { TAGCCAAGGATGACTTGCCTA-- } \\ \text { zma-miR169i-5p } & \text { TAGCCAAGGATGACTTGCCTG-- } \\ \text { zma-miR169k-5p } & \text { TAGCCAAGGATGACTTGCCTG-- } \\ \text { zma-miR169j-5p } & \text { TAGCCAAGGATGACTTGCCTG-- } \\ \text { zma-miR169d } & \text { TAGCCAAGGA-GAC-TGCCTATG } \\ \text { zma-miR169e } & \text { TAGCCAAGGA-GAC-TGCCTACG } \\ \text { zma-miR169l-5p } & \text { TAGCCAGGGATGATTTGCCTG-- } \\ \text { zma-miR169m-5p } & \text { TAGCCAAGAATGGCTTGCCTA-- } \\ \text { zma-miR169n-5p } & \text { TAGCCAAGAATGGCTTGCCTA-- } \\ \text { zma-miR169o-5p } & \text { TAGCCAAGAATGACTTGCCTA-- } \\ \text { zma-miR169p-5p } & \text { TAGCCAAGGATGACTTGCCGG-- } \\ \text { zma-miR169q-5p } & \text { TAGCCAAGAATGGCTTGCCTA-- } \\ \text { zma-miR169r-5p } & \text { CAGCCAAGGATGACTTGCCGG-- } \\ & \end{aligned}$

ata-miR398f-5p GGGTCGAACTGAGAACACATG zma-miR398a-5p GGGGCGAACTGAGAACACATG zma-miR398b-5p GGGGCGGACTGGGAACACATG

vvi-miR398b TGTGTTCTCAGGTCGCCCCTG zma-miR398a-3p TGTGTTCTCAGGTCGCCCCCG zma-miR398b-3p TGTGTTCTCAGGTCGCCCCCG

vvi-miR159c TTTGGATTGAAGGGAGCTCTA zma-miR159a-3p TTTGGATTGAAGGGAGCTCTG zma-miR159b-3p TTTGGATTGAAGGGAGCTCTG zma-miR159c-3p CTTGGATTGAAGGGAGCTCCT zma-miR159d-3p CTTGGATTGAAGGGAGCTCCT zma-miR159e-3p ATTGGTTTGAAGGGAGCTCCA zma-miR159f-3p TTTGGATTGAAGGGAGCTCTG zma-miR159g-3p TTTGGAGTGAAGGGAGTTCTG zma-miR159h-3p TTTGGAGTGAAGGGAGCTCTG zma-miR159i-3p TTTGGAGTGAAGGGAGCTCTG zma-miR159j-3p TTTGGATTGAAGGGAGCTCTG zma-miR159k-3p TTTGGATTGAAGGGAGCTCTG

$\begin{aligned} \text { osa-miR444b.1 } & ----- \text { TGTTGTCTCAAGCTTGCTGCC } \\ \text { zma-miR444a } & \text { TGCAGTTGTTGTCTCAAGCTT----- } \\ \text { zma-miR444b } & \text { TGCAGTTGTTGTCTCAAGCTT---- } \\ & \\ \text { stu-miR408b-3p } & \text {-TGCACTGCCTCTTCCCTGGCT } \\ \text { zma-miR408a } & \text { CTGCACTGCCTCTTCCCTGGC- } \\ \text { zma-miR408b-3p } & \text { CTGCACTGCCTCTTCCCTGGC- }\end{aligned}$

osa-miR444b.1 -----TGTTGTCTCAAGCTTGCTGCC zma-miR444a TGCAGTTGTTGTCTCAAGCTT-....zma-miR408b-3p CTGCACTGCCTCTTCCCTGGC-

\section{miRNAs involved in response to glyphosate treatment}

In our experiments we used RNA samples from control and glyphosate-treated plants. We collected the leaves 7 days after herbicide administration, since this is the time point when plants start to show phenotypic changes, including wilting and leaf discoloration, following exposure to glyphosate (Fig. 3). Plant miRNAs are known to be involved in adaptation to environmental stresses and in regulation of processes like growth and apoptosis, thus we were aiming at the identification of differentially expressed miRNAs related to the phenotype changes observed.

We used two technical replicate data sets obtained from both, control and glyphosate-treated samples for the analysis of expression profiles. During analysis of the sequencing data we used restrictive criteria for filtering of the miRNA expression profiling results. First, we removed redundant members of the miRNA families by filtering based on mature miRNA sequence similarity. Next, we limited the miRNA listed by minimal detection criteria (total of 10 reads in at least two cDNA libraries). After applying this procedure, we were able to observe expression of 51 representative miRNAs. Within this group we identified 13 miRNAs that revealed statistically significant expression changes ( $p$ value $<0.05$, FDR value $<0.01$ ) in glyphosate treated plants (Fig. 4). Interestingly, all of them, with one exception (miR166a-3p), were upregulated, with a $\log$ fold change in range from 1.6 up to 3.6 (Table 2).

\section{mRNA targets of differentially expressed miRNAs}

All identified differentially expressed maize miRNAs are described in the literature and their mRNA targets are well documented. However, to verify if novel miRNA family members, differentially expressed following herbicide administration, differ in functional potential from known maize miRNAs, we predicted their putative targets, based on sequence complementarity to mRNA sequences. For all differentially expressed miRNAs we identified 264 potential target transcripts, corresponding to 122 genes, of which 48 were encoding for uncharacterized proteins. Gene Ontology (GO) enrichment analysis showed that most of the proteins with known function encoded by miRNAs-targeted mRNAs were localized in nucleus and were involved in the regulation of transcription (Supporting Information File 1). The only enriched GO category not related to gene expression regulation was regulation or involvement in hydrolase activity with 17 representatives. This function was 
A

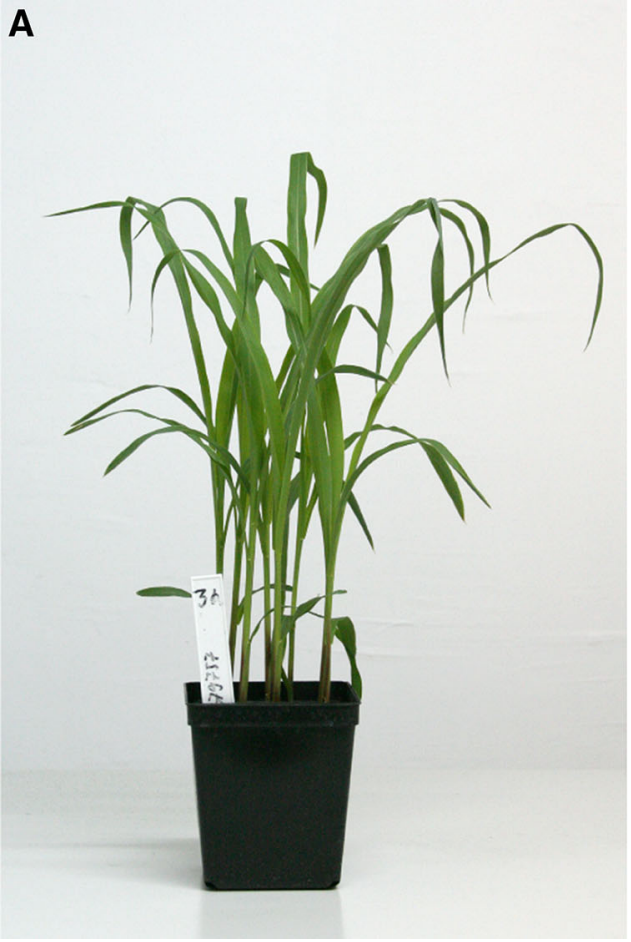

B

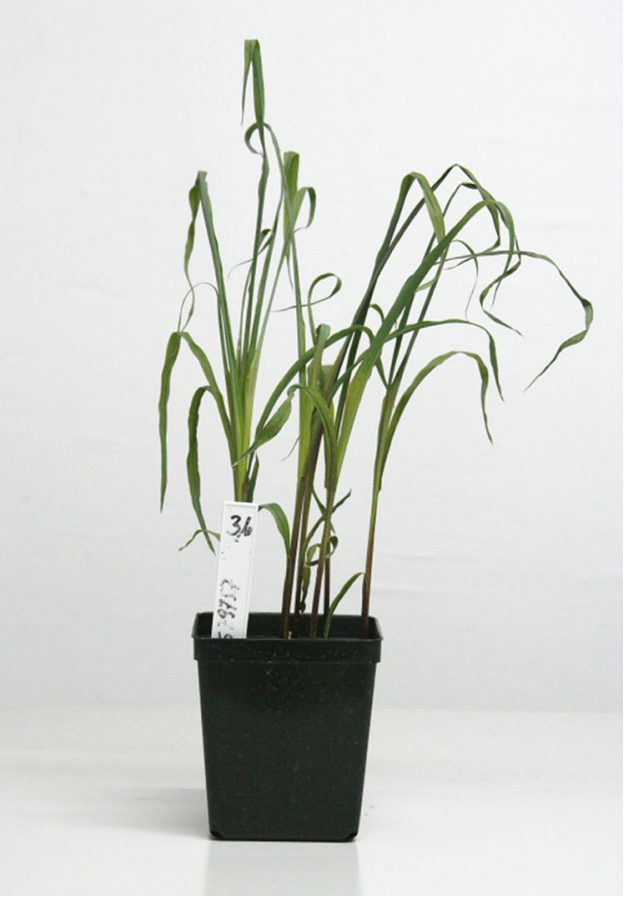

Fig. 3 Phenotype changes in maize S79757 line 7 days after glyphosate treatment. a Control, non-treated plant. b Plant sprayed with glyphosate. The change of leaf colour and wilting is clearly visible

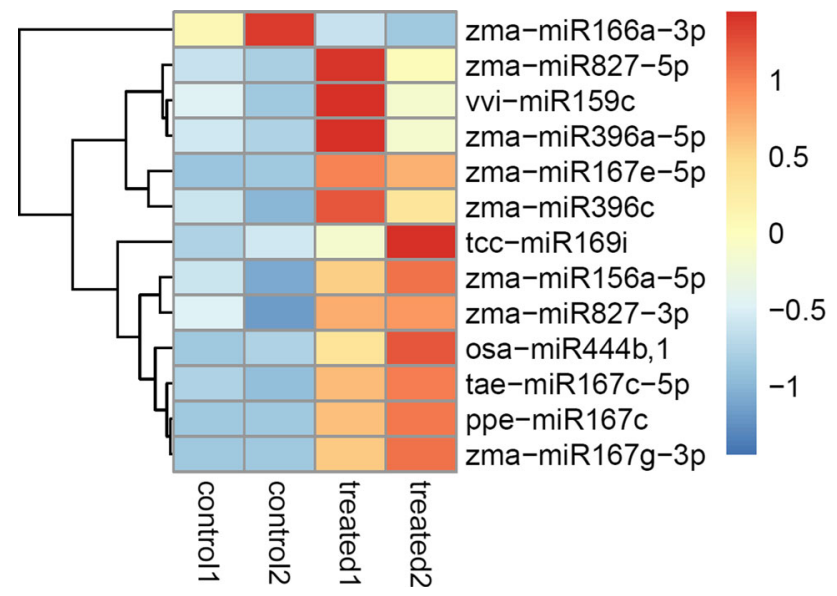

Fig. 4 Expression of miRNAs with significant differential expression upon glyphosate treatment. The clustered heatmap shows miRNA expression profiles. Individual rows representing particular miRNAs have been scaled to represent the expression level changes for different samples from the mean expression levels of given miRNA. The colour scale represents the distance (positive value and red colour stand for expression level higher than mean, negative value and blue colour represent expression level lower than mean, 0 and yellow colour is equal to mean)

assigned mostly to ATP-binding proteins. Noticeably, all predicted miRNA targets, including those for novel members of known maize miRNA families, were consistent with previous reports.
Table 2 Differentially expressed miRNAs upon glyphosate treatment

\begin{tabular}{lrrrl}
\hline & \multicolumn{1}{l}{$\log \mathrm{FC}$} & \multicolumn{1}{l}{$\log \mathrm{CPM}$} & $p$-value & FDR \\
\hline zma-miR167g-3p & $3,600,236$ & $14,30,303$ & $9.61 \mathrm{E}-16$ & $4.90 \mathrm{E}-14$ \\
ppe-miR167c & $1,814,917$ & $14,20,364$ & $7.18 \mathrm{E}-06$ & $1.83 \mathrm{E}-04$ \\
zma-miR167e-5p & $1,686,956$ & 14,5089 & $2.01 \mathrm{E}-05$ & $3.41 \mathrm{E}-04$ \\
zma-miR396c & $1,642,999$ & $15,42,785$ & $3.46 \mathrm{E}-05$ & $4.42 \mathrm{E}-04$ \\
tae-miR167c-5p & $2,364,266$ & $13,38,688$ & $6.71 \mathrm{E}-05$ & $6.85 \mathrm{E}-04$ \\
zma-miR396a-5p & $2,78,355$ & $12,92,973$ & $4.27 \mathrm{E}-04$ & $3.63 \mathrm{E}-03$ \\
zma-miR156a-5p & $1,178,679$ & $16,25,581$ & $5.49 \mathrm{E}-04$ & $4.00 \mathrm{E}-03$ \\
vvi-miR159c & $1,757,065$ & 13,9478 & $4.37 \mathrm{E}-03$ & $2.65 \mathrm{E}-02$ \\
zma-miR827-5p & $2,251,034$ & $12,08,521$ & $4.67 \mathrm{E}-03$ & $2.65 \mathrm{E}-02$
\end{tabular}

The table represents a list of miRNA differentially expressed in maize sensitive S79757 line following glyphosate spraying

$\log F C$ logarithm of expression fold change, $\log C P M$ logarithm of mean expression level in counts per million, $p$-value calculated using exact test, $F D R$ false discovery rate

\section{Discussion}

The holistic understanding of Roundup ${ }^{\circledR}$ mechanism of action is of great importance since it has been shown that glyphosate affects the growth of plants not only by inhibiting EPSPS but also through altering several crucial plant physiological processes (e.g., photosynthesis, carbon metabolism, mineral nutrition, oxidative events) (Geiger 
et al. 1986; Orcaray et al. 2012; Romero et al. 2011). Here, we focused our efforts on profiling of miRNA levels in a maize line sensitive to Roundup ${ }^{\circledR} 7$ days after herbicide administration. We identified twelve miRNAs with statistically significant upregulation in glyphosate treated plants and one downregulated (Fig. 4; Table 2). Four of them, including two isoforms observed for the first time in maize, belong to miR167 family, two are miR396 family members and two are processed from both arms of miR827 precursor. The remaining ones include miR156, miR166, and novel miR159, miR169, miR444 isoforms.

Differentially expressed members of seven out of eight microRNA families, found in our study, have previously been implicated in abiotic stress responses (Liu et al. 2012; Li et al. 2011; Kulcheski et al. 2011). In Arabidopsis (Gao et al. 2010) as well as in maize (Ding et al. 2009) miR396c is downregulated during salinity and alkali treatment. In contrast, other differentiating in our samples miRNAs, namely miR167, miR156, miR396a and miR396d, have been previously reported to be upregulated in salinity and ABA treated plants but downregulated or unchanged in plants under cold or drought stress (Jian et al. 2010; Shen et al. 2010). Interestingly, most of microRNAs that differentiate in our samples have been recently linked with senescence. It has been shown that overexpression of miR396 in transgenic Arabidopsis lines causes faster senescence of leaves when compared to wild-type plants thus suggesting its primary role in this process (Debernardi et al. 2014). miR396a-5p, newly discovered in maize, similar to miR827, is induced in Arabidopsis during senescence in leaves but repressed in siliques (Thatcher et al. 2015). Representatives of three other miRNA families (miR156, miR159, miR167) also induced upon glyphosate treatment have been implicated in senescence (Meng et al. 2012). It has been established that miR156/157 family members all target SPL transcription factors controlling development and transition from the juvenile to adult phase of the plant $(\mathrm{Wu}$ et al. 2009). It has been observed in siliques that miR156 is strongly induced in late senescence. This could in turn repress some members of SPL family of proteins (Thatcher et al. 2015). Members of remaining two miRNA families differentially expressed under glyphosate treatment, miR167 and miR159, might be involved in a mechanism of senescence-resistance through ABA-dependent pathway (Xu et al. 2014). As shown in (Xu et al. 2014) the expression levels of miR167f-3p together with miR159b and miR159a.1 were significantly lower in ageresistant rice line (N2Y6) compared to age-sensitive rice line (LYP9) that is thought to contribute to the delay of leaf senescence. Moreover, miR159, miR166 and miR167 differentiating in our study, are significantly overexpressed and functionally implicated in hybrid necrosis in wheat (Zhou et al. 2015). Both these processes (senescence and necrosis) are phenotypically closely related to changes observed in glyphosate treated maize plants.

One of the most critical processes for roundup toxicity is the penetration of glyphosate into cells. As shown in previous studies (Denis and Delrot 1993; Hetherington et al. 1998; Morin et al. 1997; Shaner 2009), glyphosate, as a phosphonate, seems to use the same phosphatespecific transport systems as inorganic phosphorus. Not only glyphosate uptake was shown to be inhibited by sodium phosphate and phosphonoformic acid, but also by PCMBS ( $p$-chromercuribenzenesulfonic acid) and DCCD ( $N, N^{\prime}$-dicyclohexylcarbodiimide) (Morin et al. 1997). It has also been demonstrated in broad bean leaves that glyphosate can be absorbed via phosphate transporter at the plasma membranes (Brecke and Duke 1980). Therefore, it was interesting to observe in maize sensitive line treated with glyphosate, the overexpression of miR827-3p and miR444, small RNAs previously shown to be directly involved in regulation of phosphorus (and nitrogen) uptake (Hackenberg et al. 2013; Lin et al. 2013; Yan et al. 2014). In Arabidopsis miR827-3p was shown to target nitrogen/phosphate balance regulator Nitrogen Limitation Adaptation (NLA, AT1g02860), a SPX-domain containing RING-type ubiquitin E3 ligase (Thatcher et al. 2015). Under Pistarvation conditions NLA is downregulated and loss of NLA causes Pi hyperaccumulation (Liu et al. 2014). Overexpression of miR827 (together with downregulation of $\mathrm{PHO} 2$ (phosphate 2) through the action of miR399) leads to degradation of PHT1 suppressor, NLA, and thus increases the levels of phosphate transporters in plasma membranes and elevated activity of Pi uptake. In rice, miR827-3p has been shown to target two genes encoding proteins containing an SPX and MFS (major facilitator superfamily) domains (Lin et al. 2010). It has been speculated that SPX-MFS proteins may be involved in intracellular or intercellular Pi transport through regulation of expression of Pi transporter (PT) genes (Yao et al. 2014) and/or Pi storage or metabolism (Lin et al. 2010). Similar to miR827-3p, monocot-specific miR444 has been shown to induce the expression of Pi transporters in rice, particularly in roots. Overexpression of miR444 leads to increased accumulation of phosphate in both roots and shoots (Lin et al. 2010). The herbicidedependent upregulation of phosphate-homeostasis-related miRNAs (miR827-3p and miR444), shown in our study, most probably triggers the increment in the level of phosphate transporters which, as a consequence, leads to increased uptake of glyphosate instead of increased 

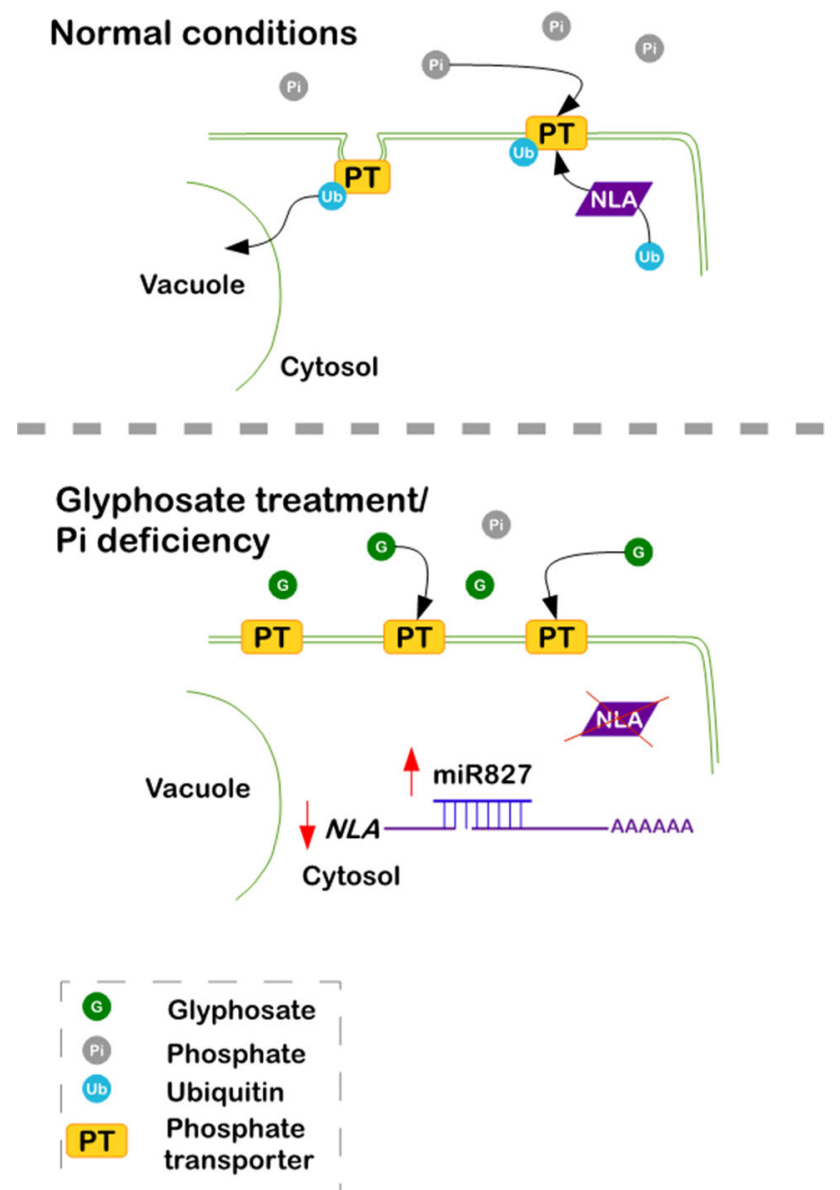

Fig. 5 miR827 role in Pi homeostasis and potentially in response to glyphosate. In Pi (inorganic phosphorus) sufficiency conditions, NLA gene (Nitrogene Limitation Adaptation) is normally expressed. NLA protein is responsible for mediating ubiquitination and degradation of membrane phosphate transporters. In Pi deficiency conditions as well as after glyphosate treatment, miR827, which targets NLA transcripts, is transcriptionally upregulated and as a consequence leads to the downregulation of NLA expression levels. As a result more phosphate transporters occur in cell membranes leading to increased $\mathrm{Pi}$ or glyphosate (depending on conditions) uptake

uptake of $\mathrm{Pi}$ and therefore disrupts the $\mathrm{Pi}$ homeostasis (Fig. 5).

The remaining miRNAs found unique upon glyphosate treatment, including miR159, miR166, miR167, miR169 and miR396 were also shown to be differentially expressed under Pi starvation in Arabidopsis, wheat, maize, soybean and white lupin (Lundmark et al. 2010; Pei et al. 2013; Zeng et al. 2010; Zhao et al. 2013; Zhu et al. 2010).

\section{Conclusions}

Our study revealed 13 miRNAs with significantly altered expression upon treatment of the plants with glyphosatebased herbicide. Most of them are known to be involved in response to abiotic stresses and senescence. Our results suggest that prominent role in mechanism of glyphosate toxicity could be associated with the disruption of phosphate transport pathways (enabling glyphosate uptake into cells by using the transporters specific for $\mathrm{Pi}$ and thus diminishing Pi uptake) and regulated through the action of miR827-3p and miR444.

Author contribution statement A.T., J.G. performed wet lab experiments; M.Ż. performed sequencing data and differential expression analysis; M.Ż., A.T., W.K., T.T. interpreted the results; M.Ż., A.T., J.G., W.K., T.T. wrote the manuscript.

Acknowledgments We thank prof. K. Adamczewski for plant breeding. Small RNA sequencing was performed at ECBiG in Poznań, Poland. Authors declare no conflict of interest. This work was supported by grants from Ministry of Science and Higher Education of the Republic of Poland no. 7690/B/P01/2011/40, National Science Center Poland nos. UMO-2011/01/D/NZ9/03631, UMO-2012/06/A/ NZ9/00125 and Ministry of Science and Higher Education of the Republic of Poland by KNOW program.

Open Access This article is distributed under the terms of the Creative Commons Attribution 4.0 International License (http://crea tivecommons.org/licenses/by/4.0/), which permits unrestricted use, distribution, and reproduction in any medium, provided you give appropriate credit to the original author(s) and the source, provide a link to the Creative Commons license, and indicate if changes were made.

\section{References}

Allu AD, Soja AM, Wu A, Szymanski J, Balazadeh S (2014) Salt stress and senescence: identification of cross-talk regulatory components. J Exp Bot 65:3993-4008. doi:10.1093/jxb/eru 173

Baev V, Naydenov M, Apostolova E et al (2010) Identification of RNA-dependent DNA-methylation regulated promoters in Arabidopsis. Plant Physiol Biochem 48:393-400. doi:10.1016/j. plaphy.2010.03.013

Brecke BJ, Duke WB (1980) Effect of glyphosate on intact bean plants (Phaseolus vulgaris L.) and isolated cells. Plant Physiol 66:656-659

Buchanan-Wollaston V, Page T, Harrison E et al (2005) Comparative transcriptome analysis reveals significant differences in gene expression and signalling pathways between developmental and dark/starvation-induced senescence in Arabidopsis. Plant $\mathrm{J}$ 42:567-585

Dai X, Zhao PX (2011) psRNATarget: a plant small RNA target analysis server. Nucleic Acids Res 39:W155-W159. doi:10. 1093/nar/gkr319

Debernardi JM, Mecchia MA, Vercruyssen L et al (2014) Posttranscriptional control of GRF transcription factors by microRNA miR396 and GIF co-activator affects leaf size and longevity. Plant J 79:413-426. doi:10.1111/tpj.12567

Denis M-H, Delrot S (1993) Carrier-mediated uptake of glyphosate in broad bean (Vicia faba) via a phosphate transporter. Physiol Plant 87:569-575. doi:10.1111/j.1399-3054.1993.tb02508.x

Ding D, Zhang L, Wang H et al (2009) Differential expression of miRNAs in response to salt stress in maize roots. Ann Bot 103:29-38. doi:10.1093/aob/mcn205 
Duke SO, Powles SB (2008) Glyphosate: a once-in-a-century herbicide. Pest Manag Sci 64:319-325. doi:10.1002/ps.1518

Friedländer MR, Mackowiak SD, Li N et al (2012) miRDeep2 accurately identifies known and hundreds of novel microRNA genes in seven animal clades. Nucleic Acids Res 40:37-52. doi:10.1093/nar/gkr688

Fu L, Niu B, Zhu Z et al (2012) CD-HIT: accelerated for clustering the next-generation sequencing data. Bioinformatics 28:3150-3152. doi:10.1093/bioinformatics/bts565

Gao P, Bai X, Yang L et al (2010) Over-expression of osa-MIR396c decreases salt and alkali stress tolerance. Planta 231:991-1001. doi:10.1007/s00425-010-1104-2

Geiger DR, Kapitan SW, Tucci MA (1986) Glyphosate inhibits photosynthesis and allocation of carbon to starch in sugar beet leaves. Plant Physiol 82:468-472

Gregersen PL, Holm PB, Krupinska K (2008) Leaf senescence and nutrient remobilisation in barley and wheat. Plant Biol (Stuttg) 10(Suppl 1):37-49. doi:10.1111/j.1438-8677.2008.00114.x

Hackenberg M, Shi B-J, Gustafson P, Langridge P (2013) Characterization of phosphorus-regulated miR399 and miR827 and their isomirs in barley under phosphorus-sufficient and phosphorus-deficient conditions. BMC Plant Biol 13:214. doi:10. 1186/1471-2229-13-214

Heap I (2015) International Survey of Herbicide Resistant Weeds. Online. Internet. Available: http://www.weedscience.com. Accessed 21 May 2015

Hetherington PR, Marshall G, Kirkwood RC, Warner JM (1998) Absorption and efflux of glyphosate by cell suspensions. J Exp Bot 49:527-533. doi:10.1093/jxb/49.320.527

Himelblau E, Amasino RM (2001) Nutrients mobilized from leaves of Arabidopsis thaliana during leaf senescence. J Plant Physiol 158:1317-1323. doi:10.1078/0176-1617-00608

Jian X, Zhang L, Li G et al (2010) Identification of novel stressregulated microRNAs from Oryza sativa L. Genomics 95:47-55. doi:10.1016/j.ygeno.2009.08.017

Jurka J (2000) Repbase update: a database and an electronic journal of repetitive elements. Trends Genet 16:418-420

Kozomara A, Griffiths-Jones S (2014) miRBase: annotating high confidence microRNAs using deep sequencing data. Nucleic Acids Res 42:D68-D73. doi:10.1093/nar/gkt1181

Kulcheski FR, de Oliveira LF, Molina LG et al (2011) Identification of novel soybean microRNAs involved in abiotic and biotic stresses. BMC Genom 12:307. doi:10.1186/1471-2164-12-307

Li T, Li H, Zhang Y-X, Liu J-Y (2011) Identification and analysis of seven $\mathrm{H}_{2} \mathrm{O}_{2}$-responsive miRNAs and 32 new miRNAs in the seedlings of rice (Oryza sativa L. ssp. indica). Nucleic Acids Res 39:2821-2833. doi:10.1093/nar/gkq1047

Lin S-I, Santi C, Jobet E et al (2010) Complex regulation of two target genes encoding SPX-MFS proteins by rice miR827 in response to phosphate starvation. Plant Cell Physiol 51:2119-2131. doi:10.1093/pcp/pcq170

Lin W-Y, Huang T-K, Chiou T-J (2013) Nitrogen limitation adaptation, a target of microRNA827, mediates degradation of plasma membrane-localized phosphate transporters to maintain phosphate homeostasis in Arabidopsis. Plant Cell 25:4061-4074. doi:10.1105/tpc.113.116012

Liu Z, Kumari S, Zhang LZ et al (2012) Characterization of miRNAs in response to short-term waterlogging in three inbred lines of Zea mays. PLoS One 7:e39786. doi:10.1371/journal.pone.0039786

Liu T-Y, Lin W-Y, Huang T-K, Chiou T-J (2014) MicroRNAmediated surveillance of phosphate transporters on the move. Trends Plant Sci 19:647-655. doi:10.1016/j.tplants.2014.06.004

Lundmark M, Kørner CJ, Nielsen TH (2010) Global analysis of microRNA in Arabidopsis in response to phosphate starvation as studied by locked nucleic acid-based microarrays. Physiol Plant 140:57-68. doi:10.1111/j.1399-3054.2010.01384.x
Martin M (2011) Cutadapt removes adapter sequences from highthroughput sequencing reads. EMBnet.journal 17: 10

Meng Y, Shao C, Ma X et al (2012) Expression-based functional investigation of the organ-specific microRNAs in Arabidopsis. PLoS One 7:e50870. doi:10.1371/journal.pone.0050870

Menne H, Köcher H (2008) HRAC Classification of Herbicides and Resistance Development. In: Modern Crop Protection Compounds. Hedelberg, Germany pp 5-26 doi:10.1002/9783527619580.ch1

Moazed D (2009) Small RNAs in transcriptional gene silencing and genome defence. Nature 457:413-420. doi:10.1038/nature07756

Morin F, Vera V, Nurit F et al (1997) Glyphosate Uptake in Catharanthus roseus Cells: role of a Phosphate Transporter. Pestic Biochem Physiol 58:13-22

Orcaray L, Zulet A, Zabalza A, Royuela M (2012) Impairment of carbon metabolism induced by the herbicide glyphosate. J Plant Physiol 169:27-33. doi:10.1016/j.jplph.2011.08.009

Pei L, Jin Z, Li K et al (2013) Identification and comparative analysis of low phosphate tolerance-associated microRNAs in two maize genotypes. Plant Physiol Biochem 70:221-234. doi:10.1016/j. plaphy.2013.05.043

Preuss S, Pikaard CS (2007) rRNA gene silencing and nucleolar dominance: insights into a chromosome-scale epigenetic on/off switch. Biochim Biophys Acta 1769:383-392

Reimand J, Arak T, Vilo J (2011) g:profiler-a web server for functional interpretation of gene lists (2011 update). Nucleic Acids Res 39:W307-W315. doi:10.1093/nar/gkr378

Robinson MD, McCarthy DJ, Smyth GK (2010) edgeR: a Bioconductor package for differential expression analysis of digital gene expression data. Bioinformatics 26:139-140. doi:10.1093/ bioinformatics/btp616

Romero DM, Ríos de Molina MC, Juárez AB (2011) Oxidative stress induced by a commercial glyphosate formulation in a tolerant strain of Chlorella kessleri. Ecotoxicol Environ Saf 74:741-747. doi:10.1016/j.ecoenv.2010.10.034

Serra A-A, Nuttens A, Larvor V et al (2013) Low environmentally relevant levels of bioactive xenobiotics and associated degradation products cause cryptic perturbations of metabolism and molecular stress responses in Arabidopsis thaliana. J Exp Bot 64:2753-2766. doi:10.1093/jxb/ert119

Shaner DL (2009) Role of Translocation as A Mechanism of Resistance to Glyphosate. Weed Sci 57:118-123. doi:10.1614/ WS-08-050.1

Shen J, Xie K, Xiong L (2010) Global expression profiling of rice microRNAs by one-tube stem-loop reverse transcription quantitative PCR revealed important roles of microRNAs in abiotic stress responses. Mol Genet Genomics 284:477-488. doi:10. 1007/s00438-010-0581-0

Thatcher SR, Burd S, Wright C et al (2015) Differential expression of miRNAs and their target genes in senescing leaves and siliques: insights from deep sequencing of small RNAs and cleaved target RNAs. Plant, Cell Environ 38:188-200. doi:10.1111/pce.12393

Van der Graaff E, Schwacke R, Schneider A et al (2006) Transcription analysis of arabidopsis membrane transporters and hormone pathways during developmental and induced leaf senescence. Plant Physiol 141:776-792

Wu G, Park MY, Conway SR et al (2009) The sequential action of miR156 and miR172 regulates developmental timing in Arabidopsis. Cell 138:750-759. doi:10.1016/j.cell.2009.06.031

Xu X, Bai H, Liu C et al (2014) Genome-wide analysis of microRNAs and their target genes related to leaf senescence of rice. PLoS One 9:e114313. doi:10.1371/journal.pone.0114313

Yan Y, Wang H, Hamera S, Chen X, Fang R (2014) miR444a has multiple functions in the rice nitrate-signaling pathway. Plant $\mathrm{J}$ 78:44-55. doi:10.1111/tpj.12446

Yao Z-F, Liang C-Y, Zhang Q et al (2014) SPX1 is an important component in the phosphorus signalling network of common 
bean regulating root growth and phosphorus homeostasis. J Exp Bot 65:3299-3310. doi:10.1093/jxb/eru183

Zeng HQ, Zhu YY, Huang SQ, Yang ZM (2010) Analysis of phosphorus-deficient responsive miRNAs and cis-elements from soybean (Glycine max L.). J Plant Physiol 167:1289-1297. doi:10.1016/j.jplph.2010.04.017

Zhao X, Liu X, Guo C et al (2013) Identification and characterization of microRNAs from wheat (Triticum aestivum L.) under phosphorus deprivation. J Plant Biochem Biotechnol 22:113-123. doi:10. 1007/s13562-012-0117-2
Zhou J, Cheng Y, Yin M et al (2015) Identification of novel miRNAs and miRNA expression profiling in wheat hybrid necrosis. PLoS One 10:e0117507. doi:10.1371/journal.pone.0117507

Zhu YY, Zeng HQ, Dong CX et al (2010) microRNA expression profiles associated with phosphorus deficiency in white lupin (Lupinus albus L.). Plant Sci 178:23-29. doi:10.1016/j.jplph. 2010.04.017 